\title{
THE NON-UNIFORM MOTION OF A THIN SMOOTH RIGID WEDGE INTO AN ELASTIC HALF-PLANE*
}

By

\author{
L. M. BROCK
}

University of Kentucky

\begin{abstract}
A two-dimensional problem of non-uniform motion by a smooth, thin, symmetric rigid wedge into the surface of an elastic half-plane is analyzed. Both the wedge starting on the surface and from an embedded position are considered. Based on the integral transforms for a more general imposed displacement, the half-plane surface and wedge path behavior yields explicit information on the various component waves appearing in the solution. Logarithmic singularities are found at the wedge apex for subsonic speeds.
\end{abstract}

1. Introduction. The splitting of an elastic material in manufacturing and mining processes may involve the motion of a relatively thin, rigid solid into the surface of the material with, ideally, little sliding friction. Here some aspects of the wave propagation associated with an idealized two-dimensional example of such motion are examined. A thin, smooth, symmetric rigid wedge moves along its symmetry plane normally into the stress-free surface of an isotropic, homogeneous, linearly elastic half-plane. The wedge speed is non-uniform and non-negative. The wedge may start on the surface or from an embedded position of static equilibrium.

The general static equilibrium position (time $t<0$ ) is shown in Fig. 1a where $h_{0} \geq 0$ is the initial penetration depth and the wedge angle is exaggerated for clarity. Actually $k \ll 1$. For convenience the variables $s=c_{1} t$ is introduced. Here $c_{1}, c_{2}$ and $\mu$ are the dilatational and rotational wave speeds and the shear modulus in the half-plane. At $t=0$ the wedge undergoes the rigid body translation $h(s) \geq h_{0}, s \geq 0$ in the positive $y$ direction where $h(s)$ is continuous, at least piecewise smooth and finite for finite $s$, with $\dot{h}(s) \geq 0, s \geq 0$. Here $\left({ }^{\circ}\right) \equiv d() / d s$. The half-plane is assumed to remain in frictionless contact with the moving wedge.

Because the wave motion is of chief interest, the superposition-related problem obtained by subtracting the initial static field from the complete solution is studied. Then, attention can be confined to the quarter-plane $x, y>0$ by introducing the boundary conditions

$$
\begin{gathered}
u=k[h(s)-y] H[h(s)-y]-k\left(h_{0}-y\right) H\left(h_{0}-y\right), \quad \sigma_{x y}=0 \\
\sigma_{x y}=0, \quad \sigma_{y}=0
\end{gathered}
$$

\footnotetext{
* Received July 26, 1978; revised version received December 8, 1979.
} 


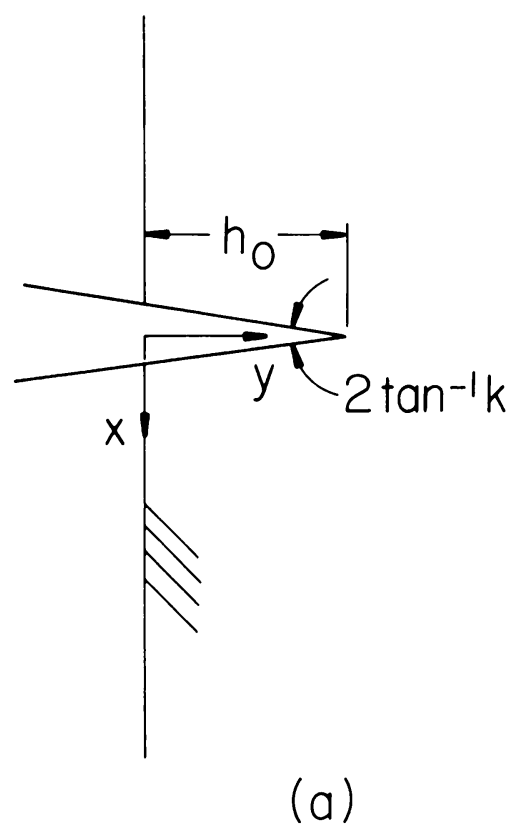

FIG. 1a. Static equilibrium position.

for $s>0$ along, respectively, the symmetry line $x=0, y>0$ and half-plane surface segment $y=0, x>0$. Here $u, v$ are the $x, y$-displacements and $H()$ is the Heaviside step function. The first term in (1.1a) imposes the normal displacement due to the wedge for $s \geq 0$ while the second term removes the displacement existing for $s<0$.

Eq. (1.1a) is an approximation valid for small displacements, frictionless contact and no separation zones. Physically, a rigid wedge travels through a half-plane by sundering the material ahead of the wedge apex. Thus, at a given location along the $y$-axis ahead of the apex, the adjacent material points on either side of the axis undergo identical displacements away from the axis, i.e. a symmetric displacement discontinuity is created. If it is assumed that the sundering occurs at the apex itself and that the displaced material points remain in contact with the wedge surfaces, then the locus of the points assumes the shape of the wedge. Therefore, if the additional assumption that the displacement component parallel to the $y$-axis is negligible compared to the normal component is made, then the normal displacement of material points which were originally located on either side of a $y$-axis location now occupied by the wedge centerline is given by (1.1a).

The neglect of the parallel component can be explicitly imposed on the problem by requiring that the velocity of the parallel component vanish. However, to avoid boundary value problem overspecification, this requirement would have to replace (1.1b). For the present problem, it is felt that the frictionless nature of the wedge motion is an important characteristic, and therefore should be explicitly imposed. Work such as [1] on the analogous problem of half-plane indentation by a rigid wedge indicates that the mixed boundary value problem arising by specifying both displacement components, i.e. 
the perfect adhesion problem, is more difficult. Moreover, in [2] the effect of neglecting the displacement component tangential to the surface of a half-plane indented by a frictionless rigid wedge was studied. It was found that the effect was negligible for very flat wedges. By analogy, it is hoped that neglecting the parallel displacement component in the present problem will be valid for a very sharp $(k \ll 1)$ wedge.

As already indicated, the material sundering may actually occur at a distance ahead of the wedge apex. Thus, a zone of separation, i.e. fracture surface, may exist such that the displaced material points come into contact with the wedge surfaces at points behind the apex. The present problem solution will indicate the effect of neglecting such a separation zone, but will also show that the zone size might be adjusted in the light of superposition.

The initial conditions on the problem are

$$
s \leq 0: \quad u, v=0
$$

for $x, y>0$. The governing differential equations for $x, y, s>0$ are

$$
m^{2}\left(\sigma_{x, x}+\sigma_{x y, y}\right)=\mu \ddot{u}, \quad m^{2}\left(\sigma_{x y, x}+\sigma_{y, y}\right)=\mu \ddot{v}
$$

where ()$_{, a} \equiv \partial() / \partial a$ and $m=c_{2} / c_{1}(0<m<1 / \sqrt{ } 2)$. The constitutive equations are

$$
\frac{m^{2}}{\mu} \sigma_{x}=u_{, x}+\left(1-2 m^{2}\right) v_{, y}, \quad \frac{m^{2}}{\mu} \sigma_{y}=v_{, y}+\left(1-2 m^{2}\right) u_{, x}, \quad \frac{1}{\mu} \sigma_{x y}=u_{, y}+v_{, x} .
$$

In addition $u, v$ should be finite in $x, y>0$ for finite $s$.

2. A general transform solution. In obtaining the transform solution for the problem it is convenient to consider, instead of (1.1a), the general condition

$$
u=U(y, s), \quad U(y, 0)=0 .
$$

Here $U(y, s)$ is an arbitrary function which is finite for finite $|y|, s$ and grows at less than exponential order as $|y| \rightarrow \infty$ or $s \rightarrow \infty$. The Laplace and Fourier sine and cosine transforms [3]

$$
\hat{g}=\int_{0}^{\infty} g(s) e^{-p s} d s, \quad g^{s}, g^{c}=\int_{0}^{\infty} g(x)(\sin p q x, \cos p q x) d x
$$

over $s>0$ and $x>0$ are employed, where $p$ is real and positive and large enough to insure convergence of $(2.2 \mathrm{a})$ while $q$ is in general complex. Operating on $(1.4 \mathrm{a}, \mathrm{b})$ with (2.2a) and, respectively, $(2.2 \mathrm{~b}, \mathrm{c})$, in view of (1.1), (1.3), (1.5) and (2.1) yields

$$
\begin{array}{r}
m^{2} \hat{u}_{, y y}^{s}-p^{2} a^{2} \hat{u}^{s}+p q\left(m^{2}-1\right) \hat{v}_{, y}^{c}=-p q \hat{U}(y), \quad a=\sqrt{ }\left(1+q^{2}\right), \\
\hat{v}_{, y y}^{c}-m^{2} p^{2} b^{2} \hat{v}^{c}+p q\left(1-m^{2}\right) \hat{u}_{, y}^{s}=\left(1-2 m^{2}\right) \frac{d \hat{U}}{d y}(y), \quad b=\sqrt{ }\left(m^{-2}+q^{2}\right)
\end{array}
$$

for $y>0$. Here $a, b$ are defined in the place cut along $\operatorname{Re}(q)=0,|\operatorname{Im}(q)|>1$ and $|\operatorname{Im}(q)| 1 / m$, respectively, so that $\operatorname{Re}(a), \operatorname{Re}(b) \geq 0$. General solutions to the coupled 
inhomogeneous equations (2.3a) and (2.4a) which are bounded for $y>0$ are

$$
\begin{aligned}
& \hat{u}^{s}=A e^{-p a y}+B e^{-p b y}-\frac{q}{a} A_{+}+B_{+}, \quad \hat{v}^{c}=\frac{a}{q} A e^{-p a y}+\frac{q}{b} B e^{-p b y}+A_{-}-\frac{q}{b} B_{-}, \quad(2.5 \mathrm{a}, \mathrm{b}) \\
& A_{ \pm}=a_{+}(y) \pm a_{-}(y), \quad B_{ \pm}=b_{+}(y) \pm b_{-}(y), \\
& 2 a_{+}=\left(2 m^{2} a^{2}-1\right) \int_{y}^{\infty} \hat{U}(z) e^{p a(y-z)} d z, \quad b_{+}=m^{2} q b \int_{y}^{\infty} \hat{U}(z) e^{p b(y-z)} d z, \\
& 2 a_{-}=\left(2 m^{2} a^{2}-1\right) \int_{-\infty}^{y} \hat{U}(z) e^{p a(z-y)} d z, \quad b_{-}=m^{2} q b \int_{-\infty}^{y} \hat{U}(z) e^{p b(z-y)} d z, \quad(2.8 \mathrm{a}, \mathrm{b})
\end{aligned}
$$

where $A$ and $B$ are arbitrary functions of $p, q$. The integration limits chosen in $A_{ \pm}, B_{ \pm}$ guarantee that the particular solution components of $\hat{u}^{s}, \hat{v}^{c}$ are bounded as $|y| \rightarrow \infty$ and prove convenient in manipulations. Operating on $(1.2 \mathrm{a}, \mathrm{b})$ with $(2.2 \mathrm{a})$ and respectively, $(2.2 \mathrm{~b}, \mathrm{c})$ in view of $(1.1),(1.3),(1.5),(2.1)$ and $(2.5)-(2.8)$ gives two equations for $A, B$. The solutions are

$$
\begin{gathered}
A=C+\frac{q}{a} a_{-}(0), \quad C=\frac{4 q^{2}}{R_{-}}\left(b^{2}+q^{2}\right) b_{+}(0)-\frac{q R_{+}}{a R_{-}} a_{+}(0), \\
B=D-b_{-}(0), \quad D=\frac{4 b q}{R_{-}}\left(b^{2}+q^{2}\right) a_{+}(0)-\frac{R_{+}}{R_{-}} b_{+}(0), \\
R_{ \pm}=4 a b q^{2} \pm\left(b^{2}+q^{2}\right)^{2} .
\end{gathered}
$$

Here $R_{-}$is a form of the Rayleigh function which has simple zeros at $q= \pm i / m_{R}$, $m_{R}=c_{R} / c_{1}$ where $c_{R}\left(0<c_{R}<c_{2}\right)$ is the Rayleigh surface wave speed. Substitution of (2.9)-(2.11) into (2.5)-(2.8) and the corresponding stress transform expressions yields

$$
\hat{u}^{s}=C e^{-p a y}+D e^{-p b y}-\frac{q}{a} C_{+}+D_{+}, \quad \hat{v}^{c}=\frac{a}{q} C e^{-p a y}+\frac{q}{b} D e^{-p b y}+C_{-}-\frac{q}{b} D_{-},
$$

$$
\begin{gathered}
\frac{1}{\mu} \hat{\sigma}_{x y}^{s}=-2 p a C e^{-p a y}-\frac{p}{b}\left(b^{2}+q^{2}\right) D e^{-p b y}-2 p q C_{-}+\frac{p}{b}\left(b^{2}+q^{2}\right) D_{-}, \\
\frac{1}{\mu} \hat{\sigma}_{y}^{c}=-\frac{p}{q}\left(b^{2}+q^{2}\right) C e^{-p a y}-2 p q D e^{-p b y}+\frac{p}{a}\left(b^{2}+q^{2}\right) C_{+}-2 p q D_{+}, \\
\frac{1}{\mu} \hat{\sigma}_{x}^{c}=\frac{p}{q}\left(2 a^{2}-m^{-2}\right) C e^{-p a y}+2 p q D e^{-p b y}-\frac{p}{a}\left(2 a^{2}-m^{-2}\right) C_{+} \\
\quad+2 p q D_{+}-4\left(1-m^{2}\right) \hat{U}(y), \\
\quad C_{ \pm}=a_{+}(y) \pm c_{-}(y), \quad D_{ \pm}=b_{+}(y) \pm d_{-}(y), \\
2 c_{-}=\left(2 m^{2} a^{2}-1\right) \int_{0}^{y} \hat{U}(z) e^{p a(z-y)} d z, \quad d_{-}=m^{2} q b \int_{0}^{y} \hat{U}(z) e^{p b(z-y)} d z .
\end{gathered}
$$

As indicated by (2.17), the contributions to $A_{ \pm}$and $B_{ \pm}$in the $z$-integration range 
$(-\infty, 0)$ appropriately cancel with, respectively, the terms $a_{-}(0)$ and $b_{-}(0)$ in $(2.9)$ and (2.10) upon recognizing that $y>0$.

Eqs. (2.12)-(2.15) are the transform solutions for the initially undisturbed quarterplane with zero surface shear stress, zero normal stress on one face and a largely arbitrary normal displacement prescribed on the other. As one solution check, the Tauberian theorems for the Fourier sine transform as defined by $(2.2 \mathrm{~b})$ require that for positive real $p$

$$
\hat{U}(y)=\lim _{|q| \rightarrow \infty} p q \hat{u}^{s} .
$$

Upon rewriting the integrals in $C_{ \pm}$and $D_{ \pm}$as

$$
\begin{gathered}
\int_{y}^{\infty} \hat{U}(z) e^{p(x)(y-z)} d z=\int_{y}^{\infty}[\hat{U}(z)-\hat{U}(y)] e^{p(x)(y-z)} d z+\frac{\hat{U}(y)}{p()}, \\
\int_{0}^{y} \hat{U}(z) e^{p(x)(z-y)} d z=\int_{0}^{y}[\hat{U}(z)-\hat{U}(y)] e^{p(x)(z-y)} d z+\frac{\hat{U}(y)}{p()}\left[1-e^{-p(y)}\right],
\end{gathered}
$$

it is readily shown that $(2.12 \mathrm{a})$ satisfies $(2.18)$, although some care must be taken for the case $y=0$.

3. The stress normal to the wedge path. Upon replacement of (2.1) with (1.1a) the stress $\sigma_{x}$ is obtained. The inversions of (2.12)-(2.14) follow the same pattern. The inverse Fourier sine and cosine transforms are defined as [3]

$$
g(x)=\frac{2 p}{\pi} \int_{i q_{0}}^{\infty+i q_{0}}\left(g^{s} \sin p q x, g^{c} \cos p q x\right) d q
$$

where the real constant $q_{0}$ is chosen so that the integration path lies in the region of analyticity for $g^{s}, g^{c}$ and convergence of $(2.2 \mathrm{~b}, \mathrm{c})$. Substitution of (1.1a) into (2.2a) and integration by parts gives

$$
\hat{U}(y)=\frac{k}{p} \int_{0}^{\infty} \dot{h}(\tau) H\left(p_{-}\right) e^{-p \tau} d \tau, \quad p \pm=h(\tau) \pm y .
$$

Eq. (2.15) shows that we can choose $q_{0}=0$ and that $\operatorname{Im}\left(\hat{\sigma}_{x}^{c}\right)=0, \operatorname{Im}(q)=0$. Therefore, substitution of (2.15) and (3.2) into (3.16) yields the Laplace transform

$$
\frac{\pi}{2 \mu k} \hat{\sigma}_{x}=\operatorname{Re} \int_{0}^{\infty} \dot{h}(\tau) e^{-p \tau} \int_{0}^{\infty}\left[\Sigma+\Sigma^{\prime}-\frac{H\left(p_{-}\right)}{m^{2} a^{2}}\right] e^{i p q x} d q d \tau
$$

where $\Sigma$ is defined as

$$
\begin{gathered}
\Sigma=K e^{-p b y}+L e^{-p|b y+a h(\tau)|}+M e^{-p b p_{+}}+N e^{-p b|p-|} \operatorname{sgn}\left(p_{-}\right), \\
L=\frac{4 b q^{2}}{a R_{-}}\left(b^{2}+q^{2}\right)\left(2 m^{2} a^{2}-1\right), \quad K=\frac{4 b q^{2}}{m^{2} a R_{-}}\left(2 m^{2}-1\right), \\
N=-2 m^{2} q^{2}, \quad M=-\frac{R_{+}}{R_{-}} N,
\end{gathered}
$$


and $\Sigma^{\prime}$ follows by interchanging $a$ and $b$ in the exponentials and substituting the corresponding primed functions of $q$ :

$$
\begin{gathered}
K^{\prime}=\frac{\left(1-2 m^{2}\right)\left(2 m^{2} a^{2}-1\right)\left(b^{2}+q^{2}\right)}{m^{4} a^{2} R_{-}}, \quad L^{\prime}=\frac{a}{b} L, \\
N^{\prime}=\frac{\left(2 m^{2} a^{2}-1\right)^{2}}{2 m^{2} a^{2}}, \quad M^{\prime}=\frac{R_{+}}{R_{-}} N^{\prime} .
\end{gathered}
$$

By the Cauchy theorem, the $q$-integration of the last term in (3.3) gives

$$
\frac{-\pi}{2 m^{2}} \int_{0}^{\infty} \dot{h}(\tau) H\left(p_{-}\right) e^{-p(\tau+x)} d \tau .
$$

The exponential in (3.7) is the Laplace transform of the Dirac delta function $\delta(s-x-\tau)$ [4]. The sifting property of the delta function can then be used to eliminate the $\tau$ integration so that the inversion of (3.7) yields

$$
\frac{-\pi}{2 m^{2}} \dot{h}(s-x) H[h(s-x)-y] .
$$

The Cagniard-deHoop [5] scheme is followed to allow inversion of the $\Sigma, \Sigma^{\prime}$-terms also by inspection. The Cauchy theorem is used to switch the original $q$-integration path to Cagniard contours, along which the exponentials in (3.4) and its $\Sigma^{\prime}$-counterpart assume the form $e^{-p n}$, where $n$ is real and positive. These contours for the $M^{\prime}, N^{\prime}, M$, and $N$-terms are readily shown to be

$$
\begin{array}{r}
r_{+}^{2} i q_{M}(n)=(y+h) \sqrt{ }\left(m^{-2} r_{+}^{2}-n^{2}\right)-n x, \quad r_{ \pm}=\sqrt{ }\left[x^{2}+(y \pm h)^{2}\right], \quad(3.9 \mathrm{a}, \mathrm{b}) \\
r_{+}^{2} q_{M}(n)=i n x+(y+h) \sqrt{ }\left(n^{2}-m^{-2} r_{+}^{2}\right), \quad r^{2} q_{N}(n)=i n x+|y-h| \sqrt{ }\left(n^{2}-m^{-2} r_{-}^{2}\right)
\end{array}
$$

for $\bar{r}_{+}<n<m^{-1} r_{+}$and $n \geq m^{-1} r_{ \pm}$, respectively, where we define

$$
\begin{aligned}
& \bar{r}_{+}=m^{-1} r_{+}, \quad x<m(y+h) / \sqrt{ }\left(1-m^{2}\right) ; \\
& \bar{r}_{+}=x+(y+h) \sqrt{ }\left(m^{-2}-1\right), \quad x>m(y+h) / \sqrt{ }\left(1-m^{2}\right)
\end{aligned}
$$

while for $n \geq r_{+}$and $n \geq r_{-}$, respectively,

$$
r_{+}^{2} q_{M}^{\prime}(n)=i n x+(y+h) \sqrt{ }\left(n^{2}-r_{+}^{2}\right), \quad r_{-}^{2} q_{N}^{\prime}(n)=i n x+|y-h| \sqrt{ }\left(n^{2}-r_{-}^{2}\right) .
$$

Here the $\tau$-dependence of $h$ is understood. Eqs. (3.10) and (3.12) indicate that the Cagniard contours include hyperbolae branches in the first quadrant of the $q$-plane with vertices at $i x / r_{ \pm}$and $i x / m r_{ \pm}$, respectively, and asymptotes $\operatorname{Arg}(q)=\tan ^{-1}(x /|y \pm h|)$. As the hyperbolae branches are traversed from the vertex to $\infty, n$ varies from $r_{ \pm}$and $m^{-1} r_{ \pm}$, respectively, to $\infty$. Eq. (3.9) shows that for $M$ an additional Cagniard contour segment may exist which follows the $\operatorname{Im}(q)$-axis from $q=i m^{-1}$ to the vertex $i x / m r_{+}$. As this path is traversed, $n$ varies from $\bar{r}_{+}$to $m^{-1} r_{+}$. It is understood that $\operatorname{Re}(q)=0^{+}$. The Cagniard contours for the $K^{\prime}$ and $K$-terms follow from those for $M^{\prime}$ and $M$ by setting $h(\tau)=0$. 
The contours for the $L^{\prime}$ and $L$-terms are defined as the $q$-solutions to, respectively, the equations

$$
a y+b z-i q x=n>0, \quad b y+a z-i q x=n>0 .
$$

It can be shown that the appropriate solutions, denoted by $q_{L}^{\prime}(n)$ and $q_{L}(n)$, respectively, give Cagniard contours which also lie in the first quadrant of the $q$-plane, for $y, h>0$ always intersect the $\operatorname{Im}(q)$-axis below the $a$-branch point $q=i$ for positive $n$ and, as $n$ increases monotonically to $\infty$, extend to infinity. These intersection, i.e. minimum, values of $n$ for $(3.13 \mathrm{a}, \mathrm{b})$ are $n_{L}^{\prime}$ and $n_{L}$, respectively, where

$$
\begin{gathered}
n_{L}^{\prime}=m^{-1} r_{1}+r_{2}, \quad n_{L}=m^{-1} r_{2}+r_{1}, \\
r_{1}=\sqrt{ }\left(x_{0}^{2}+h^{2}\right), \quad r_{2}=\sqrt{ }\left[\left(x-x_{0}\right)^{2}+y^{2}\right]
\end{gathered}
$$

and $x_{0}$ must satisfy the equation

$$
m x_{0} r_{2}, \quad m^{-1} x_{0} r_{2}=\left(x-x_{0}\right) r_{1}
$$

for $n_{L}$ and $n_{L}^{\prime}$, respectively.

In view of (3.9)-(3.15) it is readily shown that the $q$-integration of the $L$-term, for example, assumes the form

$$
\int_{n_{L}}^{\infty} K_{L}\left[q_{L}(n)\right] D q_{L}(n) e^{-p n} d n, \quad D() \equiv \partial() / \partial n .
$$

Similar results hold for the other terms. Upon substitution of these results into (3.3), the resultant exponential terms are all recognized as the Laplace transforms of $\delta(s-\tau-n)$, which can be used to eliminate the $n$-integrations. In conclusion, then, the inversion of (3.3) for $x, y, s>0$ yields

$$
\begin{gathered}
\frac{\pi}{2 \mu k} \sigma_{x}=\operatorname{Re} \int_{0}^{s} \dot{h}(\tau)\left(M_{f}+M_{r}+M_{0}\right) d \tau-\frac{\pi}{m^{2}} h(s-x) H[h(s-x)-y] H(s-x) \\
M_{f}=\operatorname{sgn}(h-y) N\left[q_{N}(s-\tau)\right] D q_{N}(s-\tau) H\left(s-\tau-m^{-1} r_{-}\right) \\
+\operatorname{sgn}(h-y) N^{\prime}\left[q_{N}^{\prime}(s-\tau)\right] D q_{N}^{\prime}(s-\tau) H\left(s-\tau-r_{-}\right) \\
M_{r}=L\left[q_{L}(s-\tau)\right] D q_{L}(s-\tau) H\left(s-\tau-n_{L}\right) \\
+M\left[q_{M}(s-\tau)\right] D q_{M}(s-\tau) H\left(s-\tau-\bar{r}_{+}\right) \\
+L^{\prime}\left[q_{L}^{\prime}(s-\tau)\right] D q_{L}^{\prime}(s-\tau) H\left(s-\tau-n_{L}^{\prime}\right) \\
+M^{\prime}\left[q_{M}^{\prime}(s-\tau)\right] D q_{M}^{\prime}(s-\tau) H\left(s-\tau-r_{+}\right) \\
M_{0}=K^{\prime}\left[q_{K}^{\prime}(s-\tau)\right] D q_{K}^{\prime}(s-\tau) H(s-\tau-r) \\
\quad+K\left[q_{K}(s-\tau)\right] D q_{K}(s-\tau) H\left(s-\tau-\bar{r}_{+}^{0}\right)
\end{gathered}
$$

where, as indicated previously, $\bar{r}_{+}^{0}$ follows from $\bar{r}_{+}$by setting $h(\tau)=0$ while $r=\sqrt{ }\left(x^{2}+y^{2}\right)$.

Some general information about the wavefront patterns in the half-planes can be obtained by examining the Heaviside functions in (3.17)-(3.20). At some instant $\tau>0$ 
suppose a source point $x=0, y=h(\tau) \geq 0$ radiates dilatational and rotational rays in the $x, y>0$ plane. For $s>\tau$ their lengths are, respectively, $s-\tau$ and $m(s-\tau)$. As defined by (3.9), $r_{-}$is the distance from the source point to a point $x, y>0$. In this light, the arguments of the Heaviside functions show that the $N^{\prime}$ and $N$-terms represent, respectively, the dilatational and rotational disturbances radiating directly from points in the displacement discontinuity region, i.e. the wedge location, for $x, y, s>0$.

In the same illustration, however, a dilatational and rotational ray from the source point can also reach a point $x, y>0$ by first traveling to the half-plane surface and then being reflected at the same angle. The total distance along such a path is, as defined by (3.9), $r_{+}$. In this light, the $M^{\prime}$ and $M$-term when $q_{M}$ is defined by (3.10) represent, respectively, the dilatational and rotational half-plane surface reflections for $x>0$ of the corresponding disturbance generated by points in the displacement discontinuity region.

When a dilatational ray reaches the half-plane surface from the source point, the stress-free conditions require that a reflected rotational ray also be generated [6]. Moreover, this reflected ray will not leave the surface at the same angle as the incident ray. It can be shown that Eqs. (3.14) and (3.15b) give the elapsed time $\left(\times c_{1}\right) n_{L}$ between the generation of a source point dilatational ray and the receiving of the rotational reflection at $x, y>0$. Here $x=x_{0}$ is the point on the half-plane surface reached by the dilatational ray. In this view, the $L$-term represents the rotational wave disturbances generated by reflection of the dilatational waves traveling from points in the displacement discontinuity region. By analogy, the $L$-term represents the dilatational waves arising from the rotational wave reflections.

When $q_{M}$ is defined by (3.9), $m \bar{r}_{+}<m(s-t)<r_{+}$and $x>m(y+h) / \sqrt{ }\left(1-m^{2}\right)$. In terms of the illustration, it can now be seen that these relations confine $x, y>0$ within a region bounded by a circular wavefront of radius $m(s-\tau)$ centered at the image source point $y=-h(\tau)$ and a curve which is tangent to this circle and intersects the $L^{\prime}$-term wavefront at the half-plane surface for $x>0$. In this light, the $M$-term for this $q_{M}$ represents rotational waves generated along the half-plane surface by the dilatational reflections of the rotational disturbances from the wedge.

As indicated previously, the parameters and variables for the $K^{\prime}$ and $K$-terms follow from those for $M$ and $M^{\prime}$, respectively, by setting $h=0$. Therefore, the discussion for the $K^{\prime}-, K$-terms need be modified only by placing the source point at $x, y=0$. Then, the $K^{\prime}$ - and $K$-terms clearly represent, respectively, dilatational and rotational disturbances radiating from the origin $x, y=0$, i.e., the corner of the quarter-plane of mathematical interest.

The non-integral term in (3.17) represents the propagation of a dilatational wave induced by the discontinuities along $x=0$ normally into the quarter-plane $x>0$. The argument of the term shows that a wedge which instantaneously attains a finite speed, i.e. $\dot{h}(0) \neq 0$, produces a stress jump at the wavefront.

In summary, then, non-superscripted and superscripted terms in (3.17) represent, respectively, rotational and dilatational signals arriving at a location $x, y>0$ due to the radiation of cylindrical waves from points in the displacement discontinuity region. The $M_{f}$, or fundamental, term represents signals received directly from the points while the $M_{r}$, or reflected, term represents the signals received upon reflection by the half-plane surface. Finally, the $M_{0}$ term represents signals received from a special point, the origin $x, y=0$.

As should be expected, if $\dot{h}$ exceeds $m$ at some $\tau$, then for $s>\tau$ a rotational Mach 


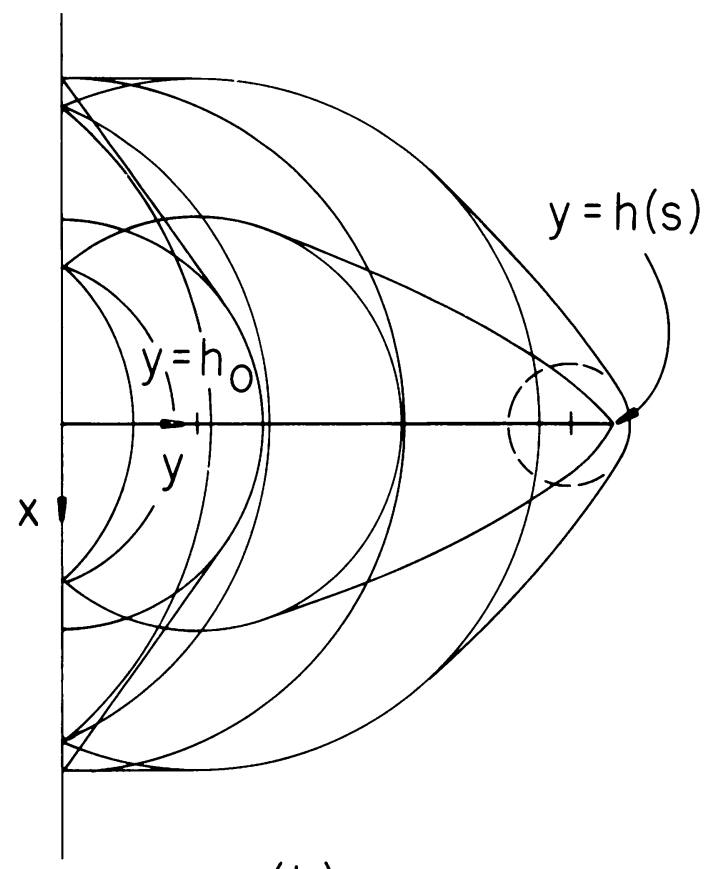

(b)

FIG. 1b. Wavefront pattern for moving wedge.

wavefront associated with the $M_{f}$-term $N$ is generated which runs from the wedge apex to a point of tangency with the locus $x^{2}+[y-h(\tau)]^{2}=m^{2}(s-\tau)^{2}$ and is defined by

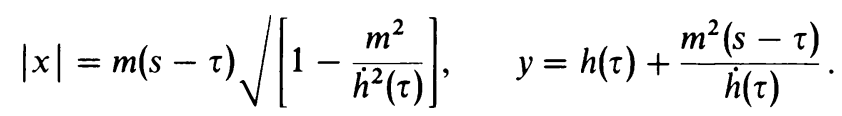

If, at some $\tau^{\prime}>\tau, \dot{h}$ drops below $m$, then for $s>\tau^{\prime}$ the Mach wavefront separates from the wedge apex to become tangent to the locus $x^{2}+\left[y-h\left(\tau^{\prime}\right)\right]^{2}=m^{2}\left(s-\tau^{\prime}\right)^{2}$. Similar observations hold for the dilatational Mach wave, where $m=1$. In Fig. $1 \mathrm{~b}$ the wavefront patterns involving Mach waves for a case of non-uniform $h$ are shown. The dashed circle indicates the locus generated by dilatational Mach wave separation; i.e., the wedge speed has dropped below the dilatational wave speed.

4. Some further observations. Along the wedge path $x=0$ (3.17) greatly simplifies because $M_{f}, M_{r}$, and $M_{0}$ are all purely real and

$$
\begin{aligned}
& q_{K}(n)=\sqrt{ }\left(\frac{n^{2}}{y^{2}}-\frac{1}{m^{2}}\right), \quad q_{M}(n)=\sqrt{ }\left(\frac{n^{2}}{p_{+}^{2}}-\frac{1}{m^{2}}\right), \quad q_{N}(n)=\sqrt{\left(\frac{n^{2}}{p_{-}^{2}}-\frac{1}{m^{2}}\right)}, \\
& q_{L}^{2}(n)=\frac{[n h(\tau)-y X]^{2}}{p_{+}^{2} p_{-}^{2}}-1=\frac{[n y-h(\tau) X]^{2}}{p_{+}^{2} p_{-}^{2}}-\frac{1}{m^{2}}, \\
& X=\sqrt{\left[n^{2}+\left(1-\frac{1}{m^{2}}\right) p_{+} p_{-}\right], \quad r^{\prime}=h(\tau)+\frac{y}{m},}
\end{aligned}
$$


where $n \geq y / m, n \geq p_{+} / m, n \geq\left|p_{-}\right| / m$ and $n \geq r^{\prime}$, respectively. For these $n$-ranges the corresponding functions and the radicals $a, b$ are real and positive. Thus, the Cagniard contours become simply parameterizations of the original $q$-integration path. The $\tau$ integration paths in (3.17) can now be represented as vertical lines in the $y \tau$-plane within regions bounded by $\tau=0, y=0$ and curves defined by the Heaviside functions appearing in the integrals. Typical paths for which $\dot{h}(s)<m$ but $\dot{h}>m$ for some $0<\tau<s$ are shown in Fig. 2. The regions also represent cross-sections at the plane $x=0$ through the volumes generated by component solution waves in $x y \tau$-space for a given $s>0$. In this light Fig. 2 confirms the observation that the $K$ - and $N$-integrals in (3.17) describe the rotational cylindrical waves radiating from, respectively, the wedge/half-plane surface intersection point $x, y=0$ and the wedge apex $x=0, y=h(s)$. The $y>m s+h_{0}$ "bulge" for the $N$-diagram indicates the rotational Mach waves radiating from the wedge apex after $\dot{h}>m$. One bounding curve slope vanishes when $\dot{h}(\tau)=m$. Similarly, Fig. 2 shows that the $L, M$-integrals do indeed describe the rotational component of the reflections from $y=0$ of, respectively, dilatational and rotational waves.

By analogy, the superscripted Cagniard contours follow from (4.1)-(4.3) by interchanging $m$ and 1 and the superscripted terms are the dilatational wave components of $\sigma_{x}$. Diagrams such as Fig. 2 can be drawn for these terms and observations strictly

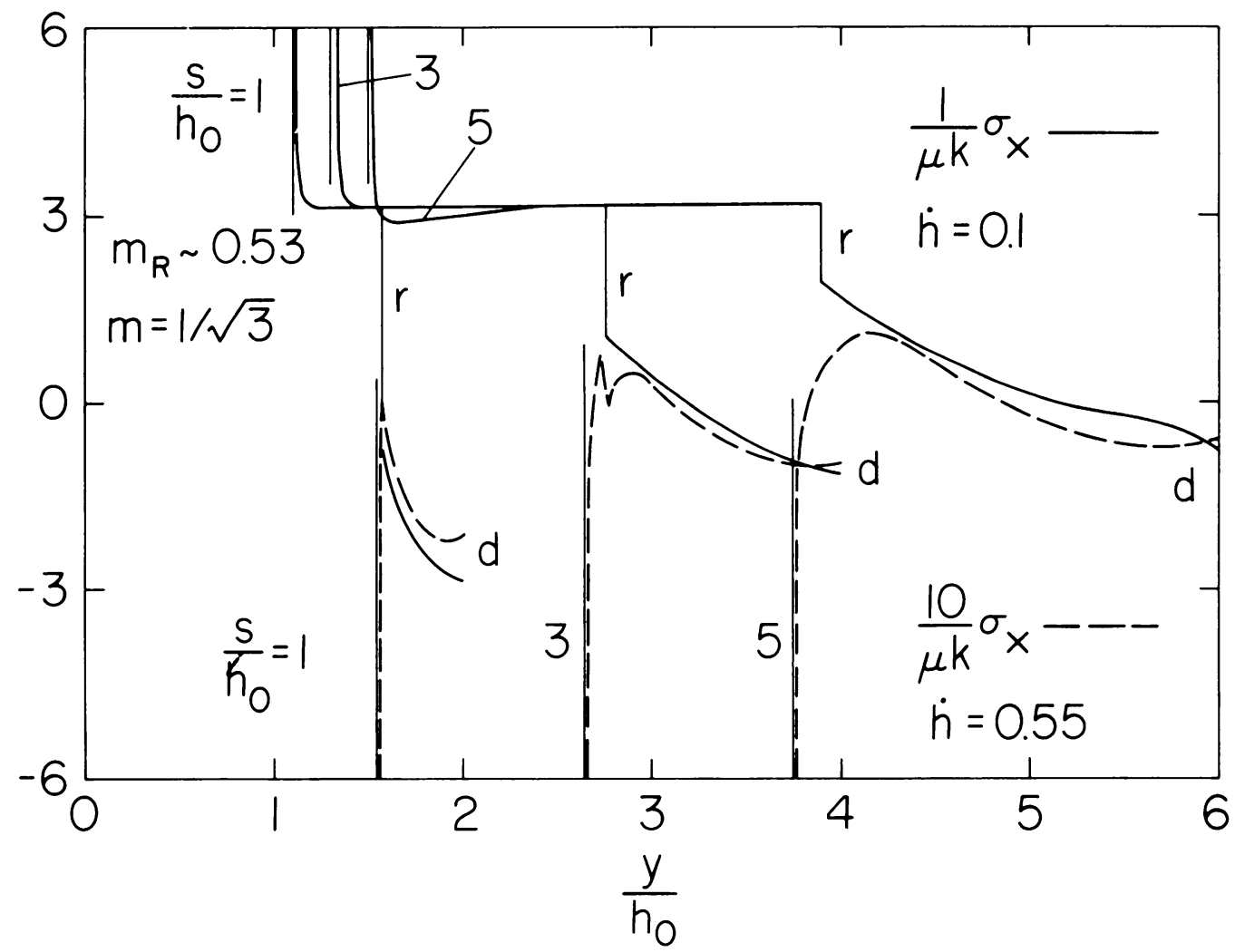

FIG. 2. Integration paths for $x=0$. 
analogous to those above made. The remaining term in (3.17) describes dilatational plane waves traveling normally away from the initial wedge slot in the region $x \leq s, 0 \leq y \leq h_{0}$ and waves moving away from the newly created wedge slot in the tapering region $x<s$, $h_{0} \leq y \leq h(s-x)$. Thus, the cylindrical waves are not the only disturbances associated with the moving wedge apex.

The previous comments indicate that no Rayleigh surface waves and no rotational waves generated by faster-traveling dilatational waves (head waves) are associated with the wedge path $x=0$. This result is not surprising in view of the nature of the boundary conditions (1.1).

Further study shows that singular behavior occurs at the wedge apex. The $M_{f}$-contribution to (3.17) for $x=0$ can be written as

$$
\begin{aligned}
& \int_{0}^{\infty} \frac{\dot{h}(\tau)(s-\tau)}{p_{-}} \mid N^{\prime}\left[q_{N}^{\prime}(s-\tau)\right] \frac{H\left(s-\tau-\left|p_{-}\right|\right)}{\sqrt{ }\left[(s-\tau)^{2}-p_{-}^{2}\right]} \\
& +N\left[q_{N}(s-\tau)\right] \frac{H\left(s-\tau-m^{-1}\left|p_{-}\right|\right) \mid}{\sqrt{ }\left[(s-\tau)^{2}-m^{-2} p_{-}^{2}\right]} \mid d \tau .
\end{aligned}
$$

By following the procedure of [7] it can be shown that (4.4) causes

$$
\frac{1}{\mu k} \sigma_{x} \sim \frac{-m^{2} R[\dot{h}(s)]}{\pi \dot{h}^{2}(s) \alpha[\dot{h}(s)]} \ln |y-h(s)|+\cdots
$$

for $\dot{h}(s)<m$ when $x=0, y \rightarrow h(s), s>0$. Here

$$
R(q)=4 \alpha(q) \beta(q)-\left(2-q^{2} / m^{2}\right)^{2}, \quad \alpha(q)=\sqrt{ }\left(1-q^{2}\right), \quad \beta(q)=\sqrt{ }\left(1-q^{2} / m^{2}\right)
$$

where $R$ is a form of the Rayleigh function with simple zeros at $q= \pm m_{R}$. Eq. (4.5) is identical to the expression obtained in [7] for the stress normal to the plane of a semiinfinite wedge moving in an unbounded solid. As in [7], it is readily shown that the $\beta$-component of (4.5) vanishes when $\dot{h}(s) \geq m$ while the $\alpha$-component behaves as $O\left(\varepsilon^{-1 / 2}\right)$, $\varepsilon \rightarrow 0$ when $\dot{h}(s) \rightarrow 1^{-}$and vanishes for $\dot{h}(s)>1$. Thus, the singularity vanishes when the wedge speed is supersonic. Moreover, $R$ changes from positive to negative as $q$ exceeds $m_{R}$. Therefore (4.5) indicates that $\sigma_{x}$ at the wedge apex is tensile at sub-Rayleigh wave speeds but compressive when that speed is exceeded.

The quantity $\sigma_{x}$ for $x=0, y>h(s)$ plays the role of a "splitting stress" in the problem. Some insight into its behavior can be obtained from Fig. 3 for the case of a wedge starting from an embedded depth $y=h_{0}>0$. There this stress is plotted against $y / h_{0}$ for the constant extension speeds $\dot{h}=0.1$ and $\dot{h}=0.55$ and various values of $s / h_{0}$. We choose the typical value $m=1 / \sqrt{ } 3$ so that the first speed is subcritical while the second lies between the Rayleigh and rotational wave speeds. Fig. 3 shows the logarithmic singularity. The lack of both temporal and spatial variation, other than the singularity, in the splitting stress near the apex is noted. Moreover, the $\dot{h}(\tau)$-factor in (3.17) and the magnitudes of the normalized quantities in Fig. 3 suggest that for this linear analysis to be valid, both $\dot{h}$ and $k$ should be very small compared to 1 . Finally, the discontinuities at the rotational and dilatational wavefronts, coming from the wedge apex and denoted by $r$ and $d$, respectively, are to be noted in Fig. 3. However, the rotational discontinuity in the $\dot{h}=0.55$ case appears to vanish as $s$ increases. 
L. M. BROCK
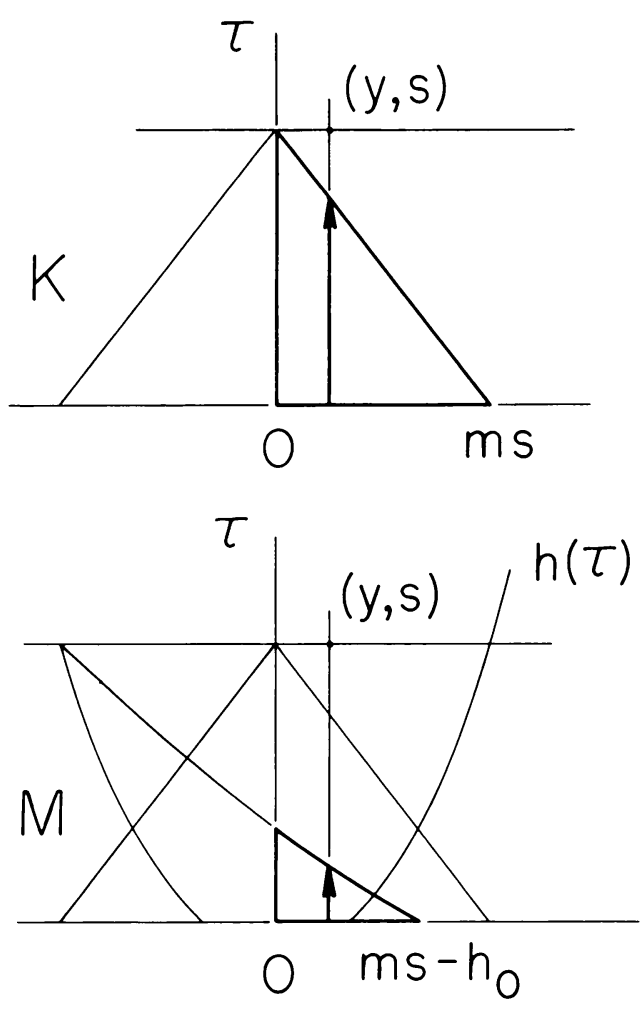
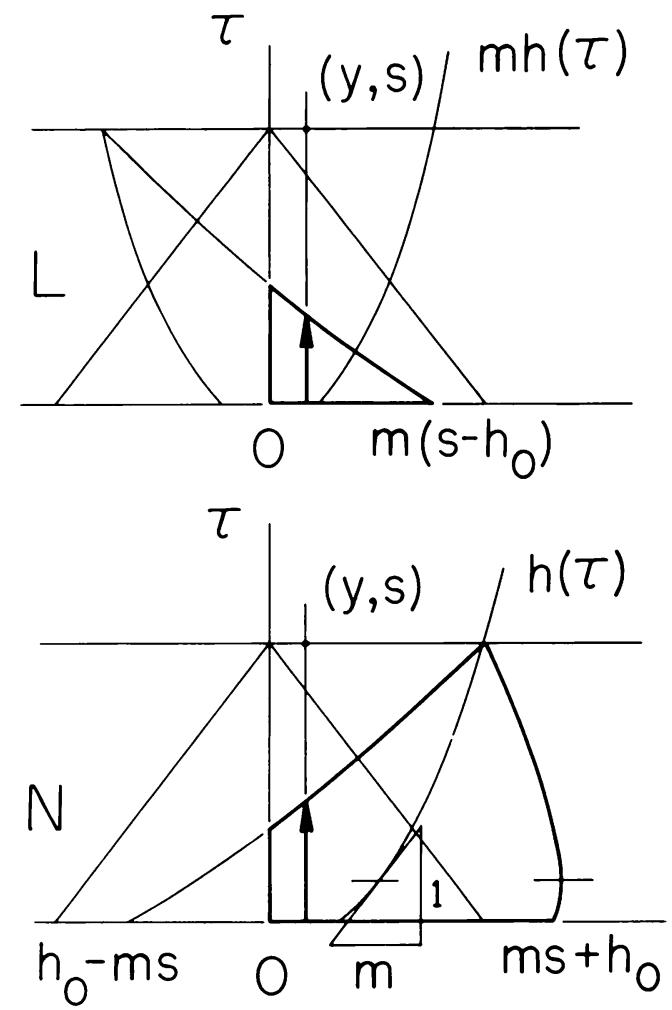

Fig. 3. Normal stress ahead of wedge apex.

5. In-plane velocity for $y=0$. For illustration the particle velocity $\dot{u}$ for $y=0$ is examined. The inversion scheme for $\dot{u}$, and for $(2.12 \mathrm{~b})-(2.15)$ as well, is similar to that of Sec. 3. The results for $x, s>0$ are

$$
\begin{aligned}
\frac{\pi}{2 k} \dot{u}= & \int_{0}^{\infty} \dot{h}(\tau) F\left(\frac{s-\tau}{x}\right) H(s-\tau-x) H\left(\tau-s+\frac{x}{m}\right) \frac{d \tau}{x}+G \dot{h}\left(s-\frac{x}{m_{R}}\right) H\left(s-\frac{x}{m_{R}}\right) \\
& +\operatorname{Im} \int_{0}^{\infty} \dot{h}(\tau) P\left[q_{P}^{+}(s-\tau)\right] D q_{P}^{+}(s-\tau) H(s-\tau-r) d \tau \\
& +\operatorname{Im} \int_{0}^{\infty} \dot{h}(\tau) Q\left[q_{Q}^{+}(s-\tau)\right] D q_{Q}^{+}(s-\tau) H\left(s-\tau-\frac{r}{m}\right) d \tau \\
& +\int_{0}^{\infty} \dot{h}(\tau) \bar{Q}[\bar{q}(s-\tau)] D \bar{q}(s-\tau) H\left(\tau-s+\frac{r}{m}\right) H\left(s-\tau-r^{\prime}\right) H(x-\bar{r}) d \tau \\
P=\frac{-2 b q}{a R_{-}}\left(2 a^{2}-m^{-2}\right), \quad Q=\frac{2 q}{R_{-}}\left(b^{2}+q^{2}\right), \quad \bar{Q}=\frac{8 c b(i q) q^{3}}{\left|R_{-}(i q)\right|^{2}}\left(d^{2}+q^{2}\right) & \\
c & =\sqrt{ }\left(q^{2}-1\right), \quad d=\sqrt{ }\left(q^{2}-m^{-2}\right) \\
F & =\frac{2 q\left(d^{2}+q^{2}\right)}{\left|R_{-}(i q)\right|^{2}} b(i q) \frac{1}{c m^{2}}\left(2-\frac{1}{m^{2}}\right)
\end{aligned}
$$




$$
G=\frac{\pi}{2}\left[\frac{-c\left(d^{2}+q^{2}\right)+d\left(2 c^{2}+m^{-2}\right)}{-2 c d\left(d^{2}+q^{2}\right)+2 c^{2} d^{2}+q^{2}\left(c^{2}+d^{2}\right)}\right] \quad\left(q=1 / m_{R}\right) .
$$

In (5.1) the Cagniard functions $q_{\bar{Q}}^{+}$and $\bar{q}$ are

$$
r^{2} q_{\bar{Q}}^{+}(n)=\operatorname{inx} \pm h(\tau) \sqrt{ }\left(n^{2}-r^{2} / m^{2}\right), \quad r^{2} \bar{q}(n)=n x-h(\tau) \sqrt{ }\left(r^{2} / m^{2}-n^{2}\right)
$$

where $n>r / m$ and $n<r / m$, respectively. In (5.1)-(5.6)

$$
r=\sqrt{ }\left[x^{2}+h^{2}(\tau)\right], \quad r^{\prime}=x+\sqrt{ }\left(\frac{1}{m^{2}}-1\right) h(\tau), \quad \bar{r}=m h(\tau) / \sqrt{ }\left(1-m^{2}\right) . \quad(5.7 \mathrm{a}-\mathrm{c})
$$

The Cagniard function $q_{\bar{P}}^{+}$follows from (5.6a) by setting $m=1$. Eq. (5.7a) defines the branch of a hyperbola in the $q$-plane with vertex $q_{\bar{Q}}^{+}=i x / m r$ and asymptotes $\arg \left(q_{\bar{Q}}^{+}\right)=$ $\pi / 2 \mp \tan ^{-1}[h(\tau) / x]$. As the hyperbola is traversed from left to right, $n$ varies from $\infty$ to $r / m$ (at the vertex) and back to $\infty$.

The $P$ - and $Q$-terms in (5.1) result from integration along the Cagniard contours defined by $q_{P}^{+}$and $q_{Q}^{+}$, respectively. For $x>\bar{r}$ the $q_{Q}$-hyperbola crosses the branch cut of the radical $a$ in $Q$ and therefore must be deformed around the branch cut portion extending below the intersection point. The $\bar{Q}$-term in $(5.1)$ is the result of integration along this deformed contour. The $F$-term appears because the Cagniard contour for one $\hat{\dot{u}}$-component simply encloses the branch cut $\operatorname{Re}(q)=0,1<\operatorname{Im}(q)<1 / m$. In addition, this component has simple poles at $q= \pm i / m_{R}$. Thus, use of the Cauchy theorem to switch the integration path results in the $G$-term as a residue.

6. Some observations on $\dot{u}$. The paths of integration indicated in (5.1) can be represented as vertical lines in bounded regions of the $x \tau$-plane. Typical paths are shown in Fig. 4 for the same situation as Fig. 2. The $P$-diagram follows from that for $Q$ by setting $m=1$. The bounded regions also represent cross-sections at the plane $y=0$ through the volumes generated by component solution waves in $x y \tau$-space for a given $s>0$. In this light Fig. 4 shows that the $F$-term in (5.1) describes the combined dilatational and rotational cylindrical wave disturbances radiating from the wedge/half-plane surface intersection point $x, y=0$. The absence of a disturbance behind the rotational wavefront is noted. The hyperbolic curve in Fig. 4 implies that the $P, Q$-terms describe, respectively, the dilatational and rotational components of the half-plane surface disturbances created by the cylindrical waves radiating from the wedge apex $x=0, y=h(s)$. The complicated diagram in Fig. 4 indicates that the $\bar{Q}$-term represents the $M$-term rotational waves described in Sec. 3 in connection with (3.9). The $G$-term in (5.1) describes the Rayleigh surface wave disturbances which radiate from $x, y=0$. Their existence follows from the stress-free nature of (1.2).

7. Brief discussion. The problem of the embedded wedge moving from a position of static equilibrium is obtained by superposition of the wave propagation problem treated here with the static solution. A study of the wave propagation problem showed that the stress normal to the wedge plane has a logarithmic singularity at the wedge apex position. This information is carried by the cylindrical waves radiating from this position and is not affected by the other waves in the problem, including reflections from the halfplane surface. The singularity disappears at supersonic wedge speeds. The singularity also indicates that the normal stress near the apex goes from tensile to compressive as the 

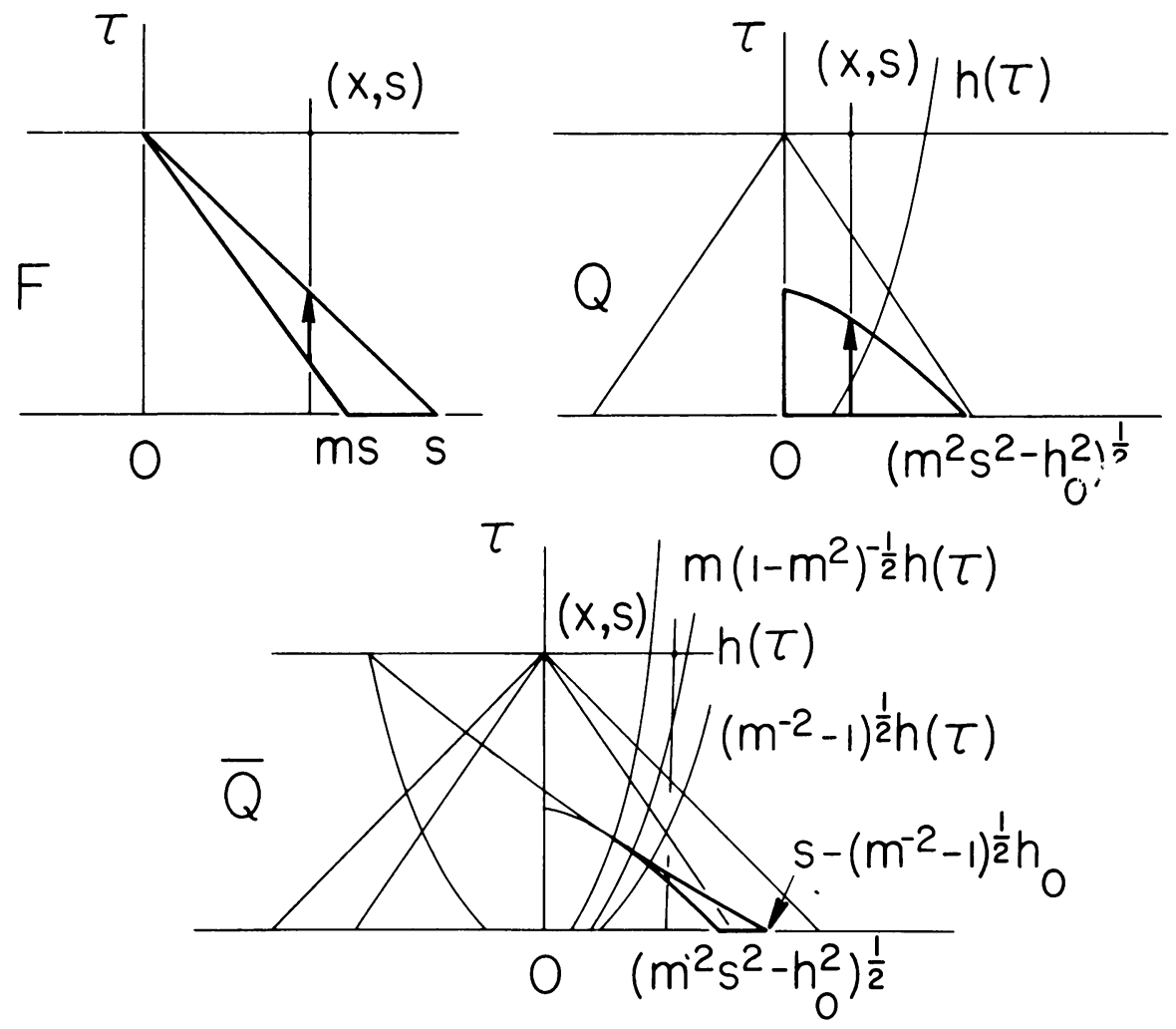

FIG. 4. Integration paths for $y=0$.

Rayleigh wave speed is exceeded. This phenomenon was noted in [7] as perhaps an implication for a limiting wedge speed. In brittle fracture theory [8] the crack edge singularities vanish when the crack attains the Rayleigh speed. Singular tensile stresses ahead of the wedge apex might imply material yielding or failure, while even finite tensile stresses on the wedge surface might imply wedge/half-plane material separation. By superposition, the actual size of the tensile zone about the apex is of course made smaller when large compressive stresses are also applied to the half-plane.

The case of the wedge starting from rest on the half-plane surface follows from the embedded wedge analysis by letting the initial wedge penetration depth vanish. Because the material is already split, the embedded wedge case provides a physical source for wedge path initiation. The surface wedge case might therefore be used as the post-surface puncture component of the half-plane splitting problem. If the initial indentation contact zone is neglected, the pre-surface puncture component could be the analysis of a normal point load on a half-plane [9]. Because the point load problem is also symmetric about the wedge path, a fully dynamic treatment involving both components could easily be handled by the present analysis. Eqs. (1.1) and (1.2) could be replaced by

$$
\begin{gathered}
u=k\left[h\left(s-s_{0}\right)-y\right] H\left[h\left(s-s_{0}\right)-y\right], \quad \sigma_{x y}=0 \\
\sigma_{x y}=0, \quad \sigma_{y}=T(s) H\left(s_{0}-s\right) \delta(x)
\end{gathered}
$$


where $h_{0}=0, T$ is one-half the point load and $s_{0}>0$ is the instant of wedge motion initiation. Its value would depend on a surface puncture criterion.

In the present analysis, the problem linearization and frictionless contact limit the possibility of a half-plane force directly opposing the wedge motion. Moreover, it can be shown that

$$
\begin{gathered}
\frac{1}{\mu k} \sigma_{y} \sim \frac{-m^{2} S[\dot{h}(s)]}{\pi \dot{h}^{2}(s) \alpha[\dot{h}(s)]} \ln |y-h(s)|+\cdots, \\
S=\left(2-q^{2} / m^{2}\right)\left[q^{2} / m^{2}-2 \alpha^{2}(q)\right]-4 \alpha(q) \beta(q)
\end{gathered}
$$

for $\dot{h}(s)<m$ when $x=0, y \rightarrow h(s), s>0$. The behavior of this singular term is similar to (4.5) except that $S>0,0<q<m$ and there is no sign reversal. Eq. (7.3) indicates, therefore, that the normal "force" on the vanishingly small area across the wedge apex is actually tensile.

In summary, the wave propagation analysis performed here was a first step in studying a potentially useful physical problem. The results will form the basis for the study of less idealized versions of the problem. The analysis was guided by the experience of [7]. In [7] an effort was made to separate the solution contributions of the two terms corresponding to those in (1.1a). In view of the more complicated wave motion, and the cumbersome solution expressions which would result, a similar effort was not made here.

It should be noted, finally, that the transform solution method used here required essentially no a priori knowledge of the complicated wave pattern. This pattern and the associated contributions, nevertheless, manifested themselves clearly in the solution. Indeed, the method has proved quite useful in treating more general non-uniformly moving displacement discontinuities [10]. However, if a knowledge of the wavefront pattern is obtained beforehand, then a dynamic similarity approach $[11,12]$ may prove to be a more efficient method of problem solution.

\section{REFERENCES}

[1] L. M. Brock, Int. J. Engng. Sci. 15, 147 (1977)

[2] L. M. Brock, Int. J. Engng. Sci. 17, 365 (1979)

[3] I. N. Sneddon, The use of integral transforms, McGraw-Hill, New York, 1972, Chaps. 2, 3

[4] I. Stakgold, Boundary value problems of mathematical physics, Vol. I, Macmillan, New York, 1967, pp. 18-24

[5] A. T. deHoop, Appl. Sci. Res. B8, 349 (1960)

[6] R. B. Lindsay, Mechanical radiation, McGraw-Hill, 1960

[7] L. M. Brock, Quart. Appl. Math. 36, 269 (1978)

[8] K. B. Broberg, Ark. Fiz. 18, 159 (1960)

[9] J. D. Achenbach, Wave propagation in elastic solids, North-Holland, Amsterdam, 1973, pp. $303-309$

[10] L. M. Brock, Int. J. Engng. Sci. 17, 1211 (1979)

[11] A. R. Robinson and J. C. Thompson, Quart. Appl. Math. 33, 215 (1975)

[12] L. M. Brock, J. Elasticity 8, 273 (1978) 